\title{
Special Issue: Emerging advances and challenges in pesticide ecotoxicology
}

\author{
Lee Shugart ${ }^{1}$
}

Published online: 20 March 2017

(C) Springer Science+Business Media New York 2017

It is the Editorial Policy of Ecotoxicology to periodically publish a Special Issue to communicate to a broad constituency of the scientific community timely information about an important topic in the environmental sciences. A Special Issue consists of assembled papers that both identify and describe the status and extent of our knowledge concerning new or existing ecotoxicological problems but also that enhances our understanding of the effects of exposure with possible linkages to ecological consequences. Within the discipline of ecotoxicology, considerable attention is directed towards the adverse impact of exposure to tailored chemicals (in particular pesticides) with an increased apprehension on the broadening effects for non-targeted organisms and habitat disruption associated with their usage. In this regard, an invitation to submit papers for a proposed Special Issue in Ecotoxicology entitled "Emerging advances and challenges in pesticide ecotoxicology" is announced.

\section{Invitation to submit manuscript abstracts for a special issue in Ecotoxicology (Springer)}

As Guest Editors of the Special Issue (SI) "Emerging advances and challenges in pesticide ecotoxicology" for the Springer's journal Ecotoxicology, we cordially invite your contribution to this SI based on your expertise in this area.

Ecotoxicology emerged following the publication of Rachel Carson's "Silent Spring" in 1962 as the

Lee Shugart

leeshugart@1rshugart.com

1 L.R. Shugart \& Associates, Oak Ridge, USA environmental branch of the field of toxicology. Its major focus was on investigating the impacts of chemicals on individuals with the goal of integrating exposure to effects on populations, communities, or ecosystems. Ecotoxicology has evolved in the past decades, resulting in several new concepts, trends, and methodologies. The ecotoxicological assessment of pesticides has also followed these new developments stimulated by the unique properties of such contaminants that are intentionally designed and released into the environment to affect specific biological targets.

This SI invites papers dealing with one or more new advances or challenges in pesticide ecotoxicology, including, but not limited to:

1. More ecological approaches, e.g. extrapolating pesticide effects from the laboratory to the field.

2. Influence of species interactions and environmental parameters on the effects of pesticides.

3. The choice of representative test species and life stages, e.g. to adhere to the new EU data requirements for ecological pesticide risk assessments.

4. Spatial-temporal extrapolation of ecotoxicological research, e.g. the influence of environmental conditions on pesticide ecotoxicity.

5. New sub-lethal test parameters, e.g. biomarkers, ecotoxicogenomics.

6. The influence of climate change on pesticide ecotoxicity.

7. Pesticide mixture toxicity.

8. Landscape and ecosystem ecotoxicology.

9. Toxicokinetics/dynamics in pesticide ecotoxicology.

10. Population and food-web modelling.

11. Getting more out of old data (e.g. meta-analysis, traitbased analysis, multivariate statistical analysis). 
The Guest Editors for this SI are Drs. Michiel Daam and Joana Luisa Pereira. Both are Associate Editors of Ecotoxicology. All contributors must submit an Abstract of their proposed paper directly to the Guest Editors for consideration. Those contributors whose Abstract has been approved will be provided specific instruction for the formal submission of their manuscript to Ecotoxicology. Please note that submission of a manuscript to the Journal at this juncture is subjected to peer-review and acceptance cannot be assured a priori.

The following tentative timeframe for publication of the SI has been approved by the Journal to ensure the contents are available to the scientific community in a timely manner:

Submission of Abstract to Guest Editors ends on June 10,2017

Submission of Approved manuscript to Journal commences July 1, 2017
Completion of Peer Review for submitted manuscripts by October 14, 2017

Submission of a revised manuscript by November 15, 2017

On-line publication of accepted manuscripts by January 1, 2018

Publication of hardcopy of SI in: Ecotoxicology volume 27(2) 2018.

Specific queries regarding this SI should be direct to the Guest Editors as listed below

Michiel Daam

Universidade Nova de Lisboa m.daam@campus.fct.unl.pt

Joana Luísa Pereira University of Aveiro jpereira@ua.pt 